Synthesis and magnetic properties of cobalt-iron/cobalt-ferrite soft/hard magnetic core/shell nanowires

This content has been downloaded from IOPscience. Please scroll down to see the full text.

Download details:

IP Address: 128.138.73.68

This content was downloaded on $30 / 04 / 2017$ at $14: 17$

Manuscript version: Accepted Manuscript

Londoño Calderón et al

To cite this article before publication: Londoño Calderón et al, 2017, Nanotechnology, at press: https://doi.org/10.1088/1361-6528/aa7010

This Accepted Manuscript is: ( 2017 IOP Publishing Ltd

During the embargo period (the 12 month period from the publication of the Version of Record of this article), the Accepted Manuscript is fully protected by copyright and cannot be reused or reposted elsewhere.

As the Version of Record of this article is going to be / has been published on a subscription basis, this Accepted Manuscript is available for reuse under a CC BY-NC-ND 3.0 licence after a 12 month embargo period.

After the embargo period, everyone is permitted to use all or part of the original content in this article for non-commercial purposes, provided that they adhere to all the terms of the licence https://creativecommons.org/licences/by-nc-nd/3.0

Although reasonable endeavours have been taken to obtain all necessary permissions from third parties to include their copyrighted content within this article, their full citation and copyright line may not be present in this Accepted Manuscript version. Before using any content from this article, please refer to the Version of Record on IOPscience once published for full citation and copyright details, as permissions will likely be required. All third party content is fully copyright protected, unless specifically stated otherwise in the figure caption in the Version of Record.

When available, you can view the Version of Record for this article at: http://iopscience.iop.org/article/10.1088/1361-6528/aa7010 


\title{
Synthesis and magnetic properties of cobalt-iron/cobalt- ferrite soft/hard magnetic core/shell nanowires
}

César Leandro Londoño-Calderón ${ }^{1}$, Oscar Moscoso-Londoño ${ }^{2,3}$, Diego Muraca ${ }^{2,4}$, Luis Arzuza $^{2,5}$, Peterson Carvalho ${ }^{2}$, Kleber Roberto Pirota ${ }^{2}$, Marcelo Knobel ${ }^{2,4}$, Laura Gabriela Pampillo ${ }^{6}$ and Ricardo Martínez-García ${ }^{7 *}$

${ }^{1}$ Institute of Polymer Technology and Nanotechnology (ITPN-CONICET), Faculty of Engineering, University of Buenos Aires, CP 1127, Buenos Aires, Argentina

${ }^{2}$ Institute of Physics "Gleb Wataghin", UNICAMP, 13083-970 Campinas SP, Brazil

${ }^{3}$ Faculty of Engineering, Autonomous University of Manizales, Antigua Estación del Ferrocarril, Manizales, Colombia

${ }^{4}$ Brazilian Nanotechnology National Laboratory (LNNano), Brazilian Center for Research in Energy and Materials (CNPEM), Zip Code 13083-970, Campinas, Sao Paulo, Brazil

${ }^{5}$ Departamento de Ciencias Naturales y Exactas, Universidad de la Costa, Calle 58 No. 55-66, Barranquilla, Colombia.

${ }^{6}$ Institute of Technology and Engineering Sciences "Hilario Fernández Long" (INTECIN-CONICET), Faculty of Engineering, University of Buenos Aires, CP 1063, Buenos Aires, Argentina

${ }^{7}$ Faculty of Natural Resources, National University of Formosa-CONICET, Campus Universitario, Modulo I, Av.

Gutnisky 3200, Formosa, Argentina

*E-mail: rmartinez@fi.uba.ar

\begin{abstract}
A straightforward method for the synthesis of $\mathrm{CoFe}_{2.7} / \mathrm{CoFe}_{2} \mathrm{O}_{4}$ core/shell nanowires is described. The proposed method starts with a conventional pulsed electrodeposition procedure on alumina nanoporous template. The obtained $\mathrm{CoFe}_{2.7}$ nanowires are released from the template and allowed to oxidize at room conditions during several weeks. The effects of partial oxidation on the structural and magnetic properties were studied by X-ray spectrometry, Magnetometry, Scanning and Transmission Electron Microscopy. The results indicate that the final nanowires are composed of $5 \mathrm{~nm}$ iron-cobalt alloy nanoparticles. Releasing the nanowires at room conditions promoted surface oxidation of the nanoparticles and creates a $\mathrm{CoFe}_{2} \mathrm{O}_{4}$ shell spinel-like structure. The shell of the nanowires avoids internal oxidation and promotes the formation of bi-magnetic soft/hard magnetic core/shell nanowires. The magnetic properties of both the initial single-phase $\mathrm{CoFe}_{2.7}$ nanowires and the final core/shell nanowires reveals that the change in the properties from the array are due to the oxidation more than effects associated to released processes (disorder and agglomeration).
\end{abstract}

Keywords: Nanowires, CoFe alloys, oxidation, core/shell nanostructures, bi-magnetic materials, soft/hard magnetic structures.

\section{Introduction}

Nanowires (Nws) are attractive materials due to their unique properties which come from their high aspect ratio (length/diameter). Moreover, shape-related effects make them of potential interest in technological and biomedical applications (spintronics, sensors, solar cells, field effect transistors, for drug and gene delivery, among others) [1-5]. The two main techniques for nanowire fabrication are lithography [6] and electrodeposition [7]. There are many reports in the literature related to the synthesis of nanowire arrays based on transition metals alloys [8,9]. Among these, those based in cobalt and iron $\left(\mathrm{CoFe}_{\mathrm{x}}\right)$ have received special attention due to their remarkable magnetic properties such as, high Curie temperature, high saturation magnetization, and large uniaxial shape anisotropy [10-12]. Surely, such properties grant them a wide range of potential applications, including data storage and medical imaging [13]. 
Previous reports have discussed the physical properties of $\mathrm{CoFe}_{\mathrm{x}}$ nanowires arrays obtained by electrodeposition [14-26]. Such studies describe mainly the relationship between Co-Fe atomic ratio and its effect on the magnetic properties. Moreover, annealing has been used in order to enhance the crystallinity and the magnetic properties of the Nws $[27,28]$. Under appropriate conditions, the thermal treatment can be used to transform $\mathrm{CoFe}_{2}$ into $\mathrm{CoFe}_{2} \mathrm{O}_{4}[29,30]$. On the other hand, Paterson et al. [31] reported core/shell $\mathrm{CoFe} / \mathrm{CoFe}_{2} \mathrm{O}_{4}$ nanowires using electrospinning technique that include several additional steps after the synthesis. Hereby a straightforward procedure for the synthesis of $\mathrm{CoFe}_{2.7} / \mathrm{CoFe}_{2} \mathrm{O}_{4}$ core/shell nanowires is proposed. In addition and in comparison to the mentioned authors, the proposed method does not require thermal treatments or controlled atmospheres. Besides, the method relies on the partial oxidation process at room conditions of the initial CoFe alloy Nws growing on alumina template. The oxidation process was development during several weeks in order to allow a growing of a thick oxide surface layer than protect the inner part of the nanowires. The method leads to bi-magnetic soft/hard magnetic core/shell nanowires. The morphological, structural and magnetic properties were studied in detail. This kind of nanostructures are promising due to their potential applications in permanent magnets, magnetic recording, microwave absorption and biomedical applications [32].

\section{Experimental details}

Nanoporous anodic aluminum oxide (AAO) templates with an average pore diameter of $29 \mathrm{~nm}$ and $3.7 \mu \mathrm{m}$ thick were prepared by two-step anodization technique [33] and subsequently used for the grow of $\mathrm{CoFe}_{2,7}$ nanowires. The electrical contact at the pore bottom was achieved by the oxide barrier layer reduction procedure [34]. The filling of the AAO's nanopores was made using a pulsed electrodeposition procedure [15] by using a homemade two-electrode electrochemical cell, with titanium as counter electrode and AAO as working electrode $(9.82 \mathrm{~mm}$ effective area).

For the synthesis of the nanowires array, a solution of $30 \mathrm{~g} / \mathrm{L} \mathrm{FeSO}{ }_{4} \cdot 7 \mathrm{H}_{2} \mathrm{O}, 15.18 \mathrm{~g} / \mathrm{L} \mathrm{CoSO}{ }_{4} \cdot 7 \mathrm{H}_{2} \mathrm{O}$, $10 \mathrm{~g} / \mathrm{L} \mathrm{H}_{3} \mathrm{BO}_{3}$, and $1 \mathrm{~g} / \mathrm{L} \mathrm{C}_{6} \mathrm{H}_{8} \mathrm{O}_{6}$ was used as electrolyte. Electrochemical deposition was performed under pulsed current of $-30 \mathrm{~mA}$ for $1 \mathrm{~ms}$, with $20 \mathrm{~ms}$ intervals. The cycle of pulses was repeated for twenty minutes at room temperature. After electrodeposition, the top side of AAO was polished in order to remove remnant clusters of deposited material. Two samples were prepared: the first one just as-prepared and the second one was dipped in $2 \mathrm{M} \mathrm{NaOH}$ solution for several hours at room temperature in order to release the nanowires. The released Nws were washed, dispersed in distilled water and collected in a silicon substrate for elemental mapping composition measurements and in a transmission electron microscopy grid. The grid and silicon substrate with the nanowires were placed at room conditions during several weeks to allow a high oxidation degree on the nanowires. For the magnetic measurements, the disperse nanowires in water were magnetically decanted in commercial plastic wrap and after dried at room conditions by several weeks.

Morphology, chemical composition and crystalline structure were analyzed by the use of Field Emission Scanning Electron Microscopy (FESEM, Zeiss Supra 40) equipped with Energy Dispersive X-ray spectrometry (EDX), and High Resolution Transmission Electron Microscopy (HRTEM, 300 $\mathrm{kV}$ with $\mathrm{LaB}_{6}$ filament, JEOL 3010). The images at the HRTEM were acquired with a Gatan 379 camera. Magnetic characterization was done using a Quantum Design-PPMS with a vibrating sample magnetometer head. The magnetic field dependence of the magnetization was measured up to $350 \mathrm{~K}$ and $3 \mathrm{~T}$. Coercive field and magnetization were measured as a function of temperature for several temperatures ranging between 5 and $350 \mathrm{~K}$. Such curves were fitted using the Marquardt-Levenberg nonlinear algorithm [35]. 


\section{Results and Discussion}

Fig. 1 shows a top view of the empty AAO template and a lateral view of the nanowires in the AAO nanocavities. AAO has a pore diameter distribution between 10 and $45 \mathrm{~nm}$ (see Fig. 1(a) inset) and a layer thickness of $3.7 \mu \mathrm{m}$. The nanopores ordering were nearly uniform and hexagonally arranged.

The AAO morphology determines the aspect ratio of the Nws array. A cross-section image of the AAO template was used to determine the nanowires length (Fig. 1(b)). Nanowires dimensions are 1.2 to $3.7 \mu \mathrm{m}$ in length and 27 to $37 \mathrm{~nm}$ in diameter. Therefore, the length/diameter ratio of nanowires goes up from 32 to 137.

The chemical composition of a single oxidized nanowire was measured by energy dispersive X-ray spectrometry (Fig. 1(c)). EDX analysis was done over many different nanowires showing an iron/cobalt ratio of 2.7/1 respectively. Intensities corresponding to carbon, oxygen, sodium and silicon were also detected. Carbon and oxygen signals are expected from manipulation of the samples at room conditions, silicon comes from the substrate used for the measurements, and the sodium appears from the dissolution process of the AAO template. Fig. 1(d) and (e) shows the elemental mapping results obtained from a single released and oxidized nanowire for the elements of interest. From the figure is clear than the distribution of $\mathrm{Fe}$ and $\mathrm{Co}$ from the electrodeposition and oxidation are homogeneous along the nanowire. Fig. 1(f) shows the nanowires aggregates after the template removal and magnetically decanted for the magnetic measurements. From the figure is clear than the releasing process promotes the formation of bundles of Nws randomly oriented.

Fig. 2(a) shows a TEM micrograph corresponding to the oxidized nanowires released from the AAO. The nanowires morphology expected from AAO geometry, was confirmed from TEM measurements. The obtained mean diameter was around $32 \mathrm{~nm}$, which closely fits the nanopore sizes of the AAO. From high resolution images a compact agglomerate of several nanoparticles (Nps), with average size is around $5 \mathrm{~nm}$ forming the nanowires can be observed (see Fig. 2(b)). Similar results were reported by other authors for Co-Fe nanowires obtained by electrodeposition $[14,18]$.

The inset of the Fig. 2(b) shows the Fast Fourier Transform (FFT) of the HR-TEM area marked with a yellow square. The nanoparticles array on the surface of the oxidized nanowires exhibit a polycrystalline structure with two different cubic phases, simple and spinel-type. The (110) reflections are associated to the simple cubic structure while the planes (220) and (311) correspond to the spinellike structure (see inset Fig. 2(b)).

To identify the simple cubic and spinel structures, Selected Area Electron Diffraction (SAED) analysis was performed on the inner (Fig. 2(c)) and the surface (Fig. 2(d)) of the nanowires. Three crystalline phases were identified: CoFe (JCPDS card No. 49-1567), $\mathrm{Co}_{3} \mathrm{Fe}_{7}$ (JCPDS card No. 481817), and one spinel-like structure at the surface, $\mathrm{CoFe}_{2} \mathrm{O}_{4}$ (JCPDS card No. 22-1086) most likely due to the oxidation process. Since the Nps on the nanowires are small in size, it is reasonable to believe that those at the surface will experience a complete oxidation $[36,37]$ and will act as a coating layer to those located at the interior of the nanowire. 


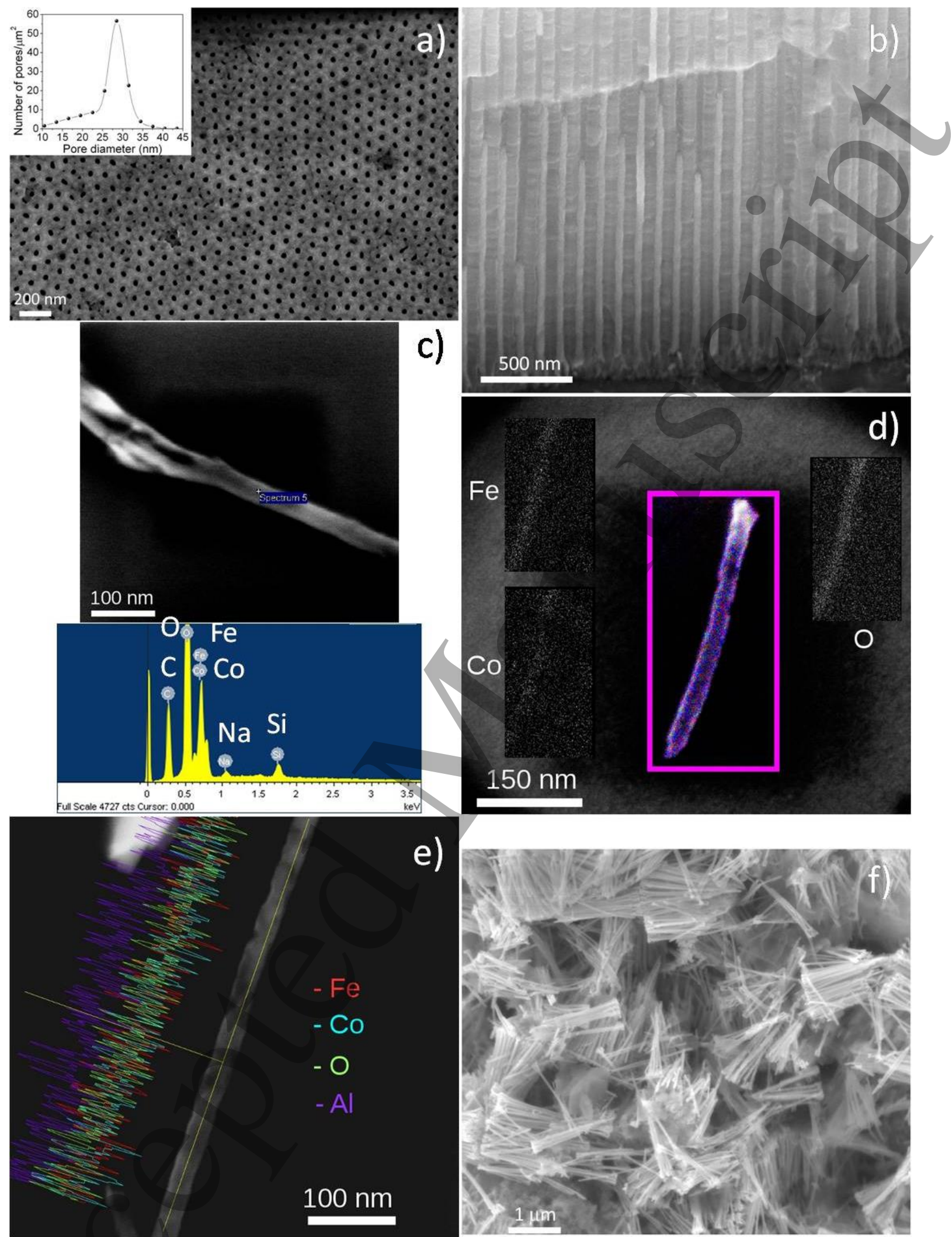

Fig. 1. FESEM micrographs of: (a) empty AAO top-view, (b) cross-sectional view of nanowires into the AAO nanocavities, (c) A single Nw released and oxidized onto silicon substrate with the corresponding EDX spectrum. Elemental mapping on (d) a single nanowire and (e) a scan along of symmetry axis of the Nw. (f) Nws aggregates after the template removal used for magnetic measurements. 
The observed crystallographic planes corresponding to the identified crystalline phases are summarized in Table 1. Both cobalt-iron alloy phases are similar from the crystallographic point of view, with the same crystalline planes (hkl) and similar interplanar distances (see Table 1). CoFe and $\mathrm{Co}_{3} \mathrm{Fe}_{7}$ give a stoichiometric average ratio of $\mathrm{Co}: \mathrm{Fe}=1: 2$, the same used in the electrolyte. Different electronegativity of cobalt and iron could be the reason for the formation of two crystalline phases during the nanowires synthesis. This difference among electronegativities generates a different deposition velocity [38] and, as result, the stoichiometry is not fixed along all the time of deposition.

Previous studies about the oxidation kinetics in iron-cobalt alloys suggest that the oxide zone thickens parabolically with time. Parabolic rate law indicates that cationic diffusion across the growing oxide layer controls the growth rate [39]. Since the nanowires exhibit crack-free oxide layers forméd by a porous-free outer layer (Fig. 2(a) and (b)), the cationic diffusion with the oxidation time is most difficult. Therefore, nanoparticles on the nanowires are essentially passivated by the thin oxide [40]. For applications at room conditions this oxide shell means that other passivating coatings are unnecessary [41]. Therefore, the growing of oxide layer during several weeks must be slow and leads to a quasi-stationary process.
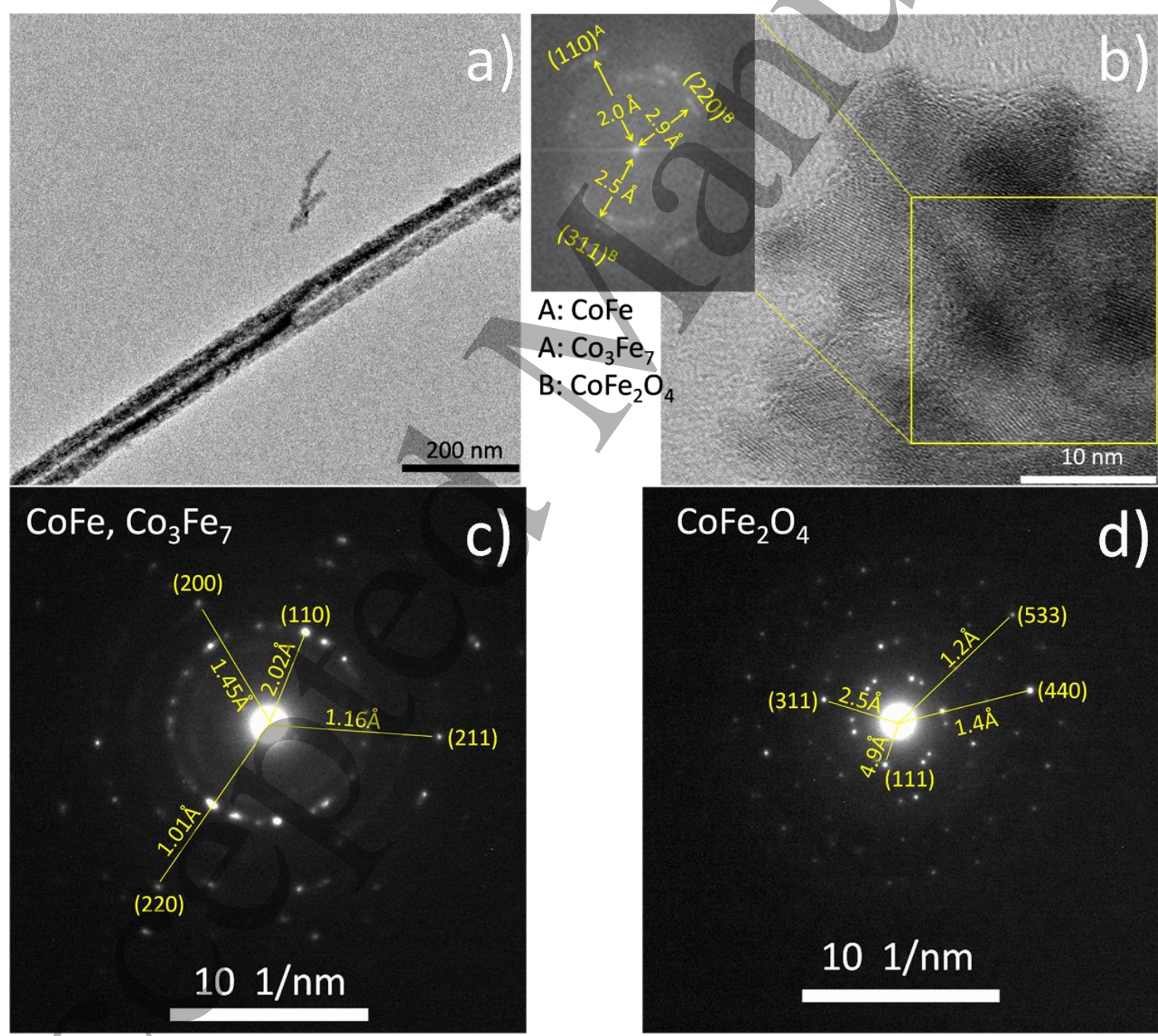

Fig. 2. (a) TEM image of the nanowires. (b) HR-TEM image of the surface of a Nw. The inset shows the FFT of the area in yellow. Selected Area Electron Diffraction (SAED) pattern of (c) inner and (d) surface of a nanowire. 
Table 1. Reported and observed planes corresponding to the crystal structures detected in nanowires. The uncertainties of the interplanar distances are shown in parentheses

\begin{tabular}{|c|c|c|c|}
\hline crystalline phase & reported (hkl) & reported d $[\AA]^{\mathrm{a}}$ & observed d [Å] \\
\hline $\mathrm{CoFe}$ & 110 & 2.0182 & $2.02(1)$ \\
\hline $\mathrm{Co}_{3} \mathrm{Fe}_{7}$ & 110 & 2.0247 & \\
\hline $\mathrm{CoFe}$ & 200 & 1.4246 & $1.45(2)$ \\
\hline $\mathrm{Co}_{3} \mathrm{Fe}_{7}$ & 200 & 1.4317 & \\
\hline $\mathrm{CoFe}$ & 211 & 1.1655 & $1.16(2)$ \\
\hline $\mathrm{Co}_{3} \mathrm{Fe}_{7}$ & 211 & 1.1692 & \\
\hline $\mathrm{CoFe}$ & 220 & 1.0094 & $1.01(1)$ \\
\hline $\mathrm{Co}_{3} \mathrm{Fe}_{7}$ & 220 & 1.0127 & \\
\hline $\mathrm{CoFe}_{2} \mathrm{O}_{4}$ & 111 & 4.8470 & $4.94(1)$ \\
\hline $\mathrm{CoFe}_{2} \mathrm{O}_{4}$ & 311 & 2.5310 & $2.58(3)$ \\
\hline $\mathrm{CoFe}_{2} \mathrm{O}_{4}$ & 440 & 1.4830 & $1.47(2)$ \\
\hline $\mathrm{CoFe}_{2} \mathrm{O}_{4}$ & 533 & 1.2798 & $1.22(1)$ \\
\hline
\end{tabular}
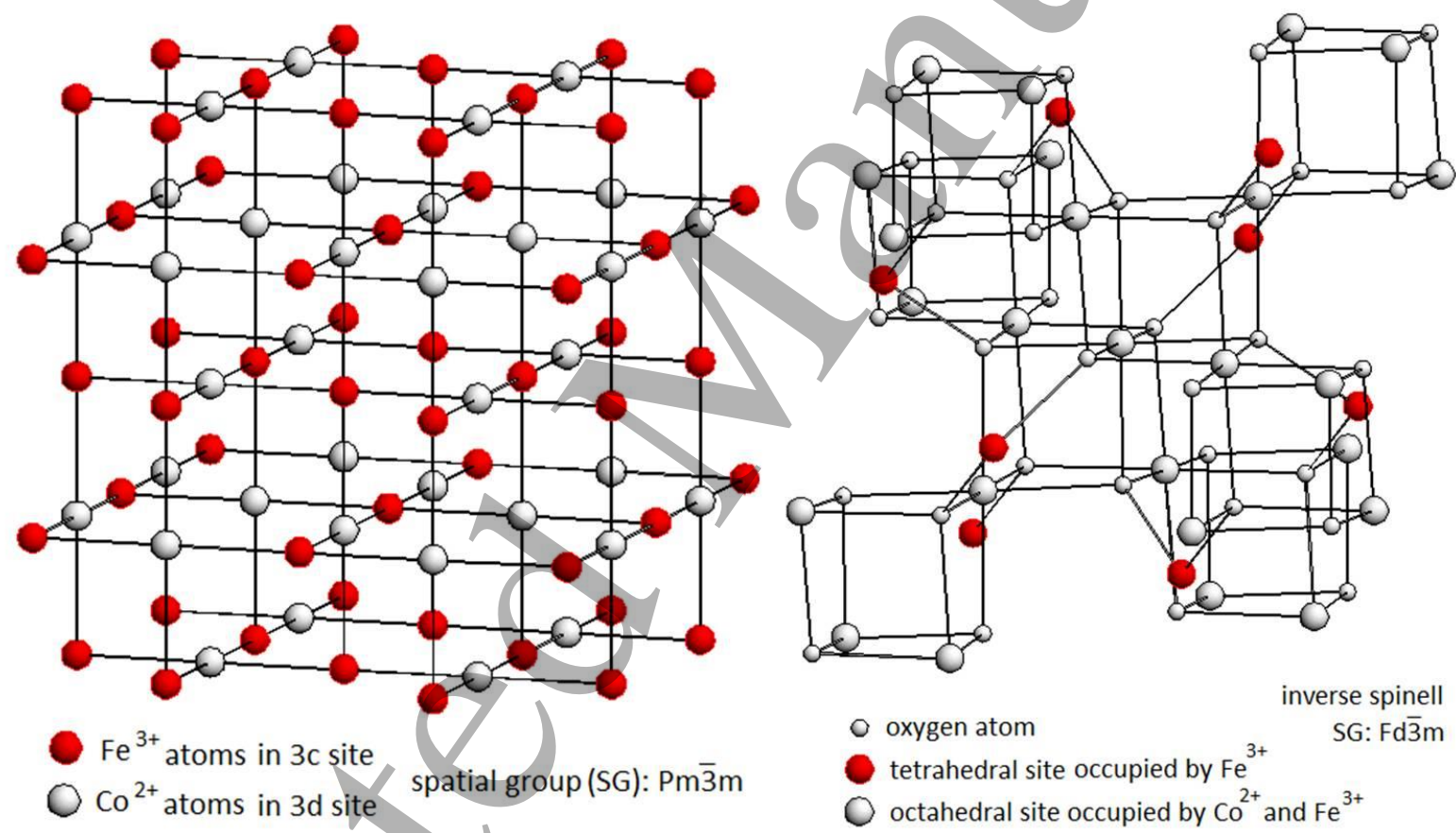

Fig. 3. Unit cells plus bonds of: $\mathrm{CoFe}$ and $\mathrm{Co}_{3} \mathrm{Fe}_{7}$ crystal structure (left), and $\mathrm{CoFe}_{2} \mathrm{O}_{4}$ spinel crystal structure (right).

On the other hand, previous reports suggest than the oxidation in iron/cobalt alloys is a selective process [39]. Where the oxidation occurrence is mainly for iron zones. At the initial stages of the oxidation there is a formation of iron rich oxide that promotes the enrichment of cobalt at the interface layer. After this initial stage, solid-state diffusion of cations and anions across the oxide scale controls the growth rate. In a simplified model for diffusion controlled oxidation, the transport proceeds by vacancies and ionizing phase boundary reactions. Iron ionizes at the metal-oxide interface and the iron ions and electrons migrate outward through the oxide layer while the ionization of oxygen takes place at the oxide-air interface. The oxygen ion diffuses inward and reacts with solute rich inner layer and 
form islands of cobalt ferrite through internal oxidation. Through theses steps the surface shell is formed and protect of oxidation the inner part of the nanowires.

The magnetic properties of nanowires depend on crystal structure of the phases as well as of the atomic interaction between atoms. For example, in magnetic alloys such as $\mathrm{CoFe}$ and $\mathrm{Co}_{3} \mathrm{Fe}_{7}$, the cobalt coordination spheres are formed by the iron atoms (see Fig. 3 left) which give place to a direct ferromagnetic exchange interaction. Moreover, on cobalt ferrite $\left(\mathrm{CoFe}_{2} \mathrm{O}_{4}\right)$, the cobalt and iron atoms are connected through an intervening oxygen atom (see Fig. 3 right) establishing a superexchangetype interaction. It is clear then, that the differences between the atomic structures of the two phases lead to complex magnetic dynamics in the $\mathrm{CoFe}_{2.7} / \mathrm{CoFe}_{2} \mathrm{O}_{4}$ core/shell nanowires.
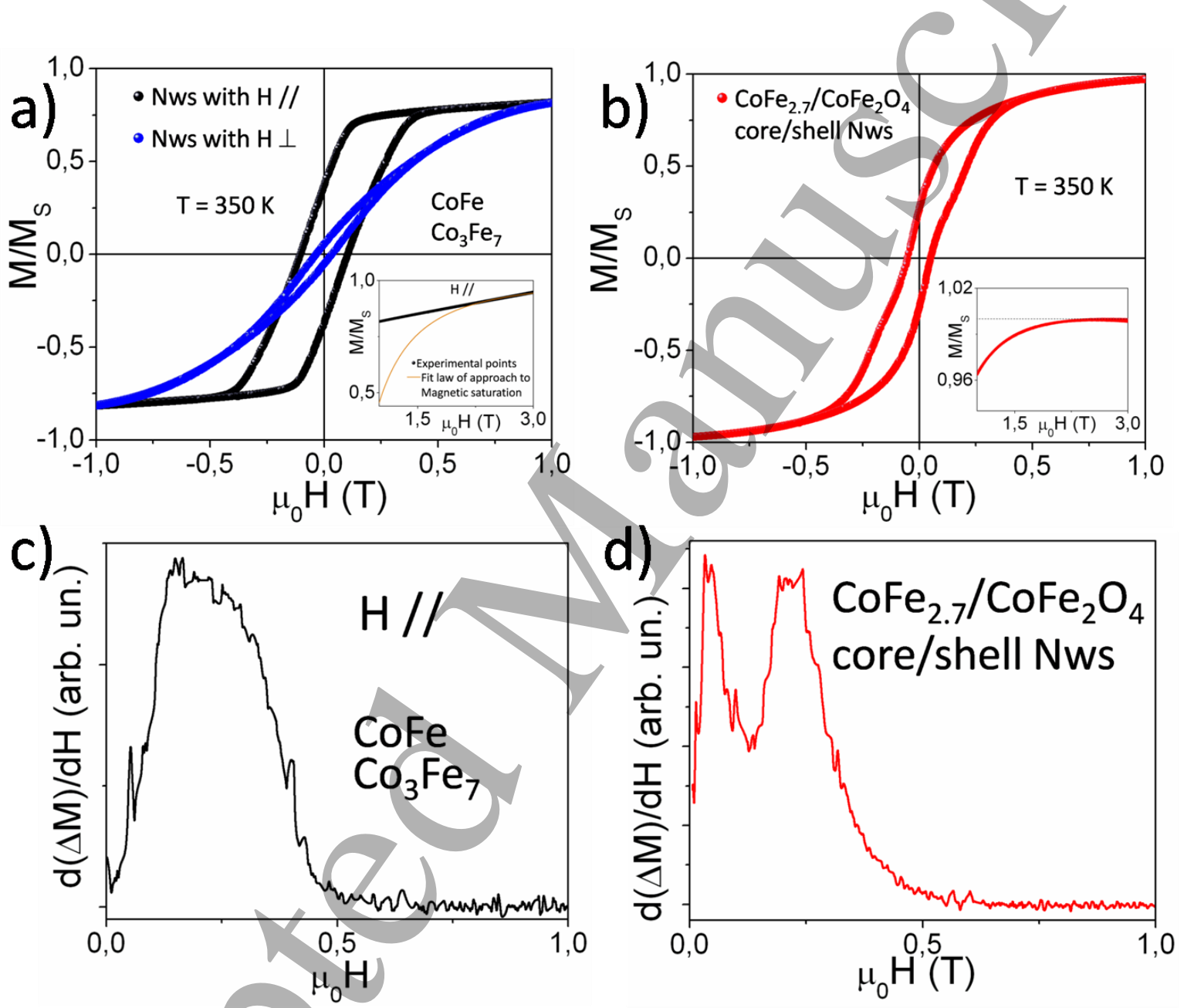

Fig. 4. Hysteresis curves at $350 \mathrm{~K}$ corresponding to: (a) nanowires into the AAO template; and (b) released and partially oxidized nanowires. The insets show the highest magnetic field zones. The first derivative of $\Delta M$ as a function of $H$ is show for (c) single-phase and (d) core/shell nanowires.

Fig. 4. shows normalized magnetization $\left(M / M_{S}\right)$ as a function of applied magnetic field $(H)$. For nanowires within the AAO template the hysteresis curves were measures with $H$ parallel and perpendicular to the Nws axis (Fig. 4(a)). The results obtained from these two measurements are fairly different. Despite that $\mathrm{CoFe}$ and $\mathrm{Co}_{3} \mathrm{Fe}_{7}$ phases have a low magnetocrystalline anisotropy [42], the high aspect ratio induces an easy magnetization axis [24,43]. For an applied magnetic field perpendicular to the nanowires axis, the magnetic saturation is reached up at higher $\mathrm{H}$ values. It is worth noting that for applied $\mathrm{H}$ parallel to the Nws axis, the values of reduced remanence 
magnetization $\left(\mathrm{M}_{\mathrm{R}} / \mathrm{M}_{\mathrm{S}}=0.37\right)$ and coercive field values $\left(\mu_{0} \mathrm{H}_{\mathrm{c}}=104 \mathrm{mT}\right)$ are in very good agreement with those reported previously $[16,18,19,25]$. However, such values and the hysteresis loops shape are very different for $\mathrm{CoFe}_{2.7} / \mathrm{CoFe}_{2} \mathrm{O}_{4}$ core/shell nanowires. The smoothed hysteresis loop for the core/shell nanowires is related with the lost of spatial order when them are released from the AAO template [44]. On the other hand, the $\mathrm{CoFe}_{2} \mathrm{O}_{4}$ layer formed during the oxidation process strongly influences the magnetization dynamic. The exchange interaction between both constituents (magnetically soft/hard) affects also the hysteresis loop [32] (see Fig. 4(b)). Such distortion result as a consequence of both magnetic phases contribution, which have different coercive field and remanence values [45], as well as the magnetic interaction among them [32]. By means of the first derivative of $\Delta M$ as a function of $H$ it is possible to determine the number of such magnetic phases [46] (Fig. 4(c) and 4(d)). As expected, just one maximum (wide peak) is observed for Nws measured into the AAO template. In this case the phases $\left(\mathrm{CoFe}\right.$ and $\left.\mathrm{Co}_{3} \mathrm{Fe}_{7}\right)$ are equivalent one to another from the chemical and structural point of view. Furthermore, $d \Delta M / d H$ vs. $H$ curve corresponding to the $\mathrm{CoFe}_{2.7} / \mathrm{CoFe}_{2} \mathrm{O}_{4}$ core/shell nanowires shows two peaks, one related to the surface while the other one is associated to the core.
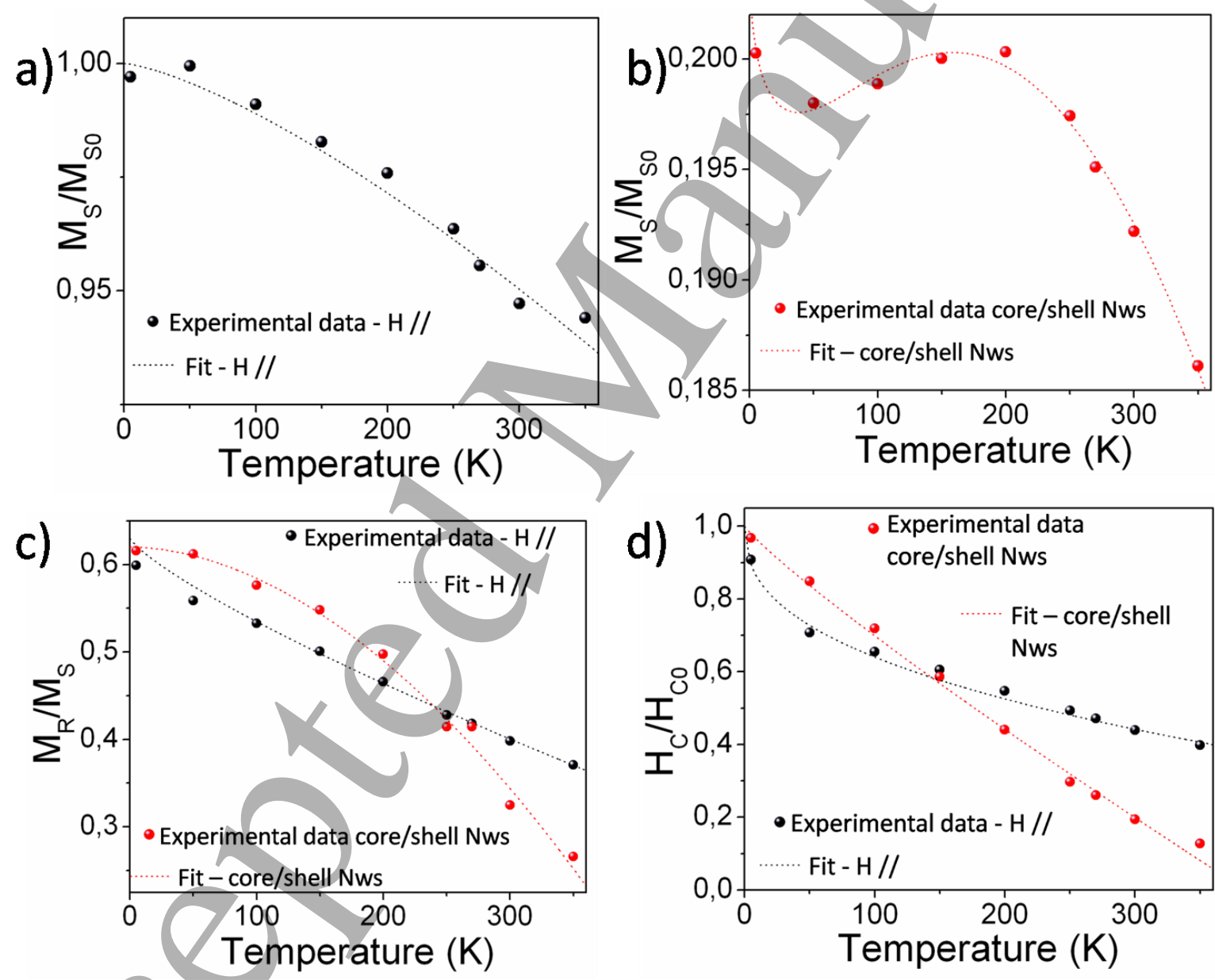

Fig. 5. Normalized magnetization as a function of the temperature for (a) nanowires within the AAO template (applied field up $3 \mathrm{~T}$ parallel to the Nws axis) and (b) core/shell nanowires. (c) Reduced remanence magnetization and (d) normalized coercive field as a function of temperature for both nanowires in the AAO template and core/shell system.

Fig. 5 shows the evolution of the normalized magnetization, remanence and coercive field as a function of temperature. For core/shell Nws all analyzed parameters $\left(M_{S}, M_{R} / M_{S}\right.$ and $\left.\mu_{0} H_{C}\right)$ exhibit 
strongest dependence within the temperature than those obtained for $\mathrm{CoFe}_{2.7}$ (these values are the lowest at high temperature). Due to oxidation, the coordination of the cobalt and iron atoms change (formed by oxygen atoms also). As a result, an indirect or superexchange interaction is established, between the two metals through oxygen atoms. Hence, two kinds of magnetic interactions coexist: a superexchange interaction at the Nws external layer (metal-oxygen-metal), and a direct exchange interaction in the nanowire core (metal-metal). The competition between these two types of magnetic interactions causes a decrease of the average module of the exchange coupling constant $(J)$. For $\mathrm{CoFe}$ and $\mathrm{Co}_{3} \mathrm{Fe}_{7}$ the module of $J$ is $69 \mathrm{meV}$ [47], and for the mixed oxide $\mathrm{CoFe}_{2} \mathrm{O}_{4}$ is $4 \mathrm{meV}$ [48]. Since $J$ is related with the spin-wave stiffness constant [47], a reduction of $J$ value enhance the magnetic properties as a function of temperature [49]. The strength of the superexchange interaction depends on the distance between atoms and on the angle formed by the interacting orbitals. Shorter distances and angles closer to $180^{\circ}$ promote the overlapping density and thus the magnetic interaction. When temperature increases the atomic movement leads to a decrease of the overlapping density and the superexchange weakens.

Table 2. Magnetic Properties: best fitting parameters corresponding to $M_{S}, M_{R} / M_{S}$, and $\mu_{0} H_{C}$ as a function of temperature obtained from equations (1), (2), (3) and (4). $B$ and $\alpha$ are the Blochs' constant and exponent, respectively. $\mu_{0} H_{C}$ and $M_{R O} / M_{S O}$ are the coercive field and reduced remanence magnetization, respectively, when $\mathrm{T} \rightarrow 0 \mathrm{~K}$. $T_{O R}$ and $T_{O C}$ are characteristic temperatures and, $\beta$ and $m$ are the exponents.

\begin{tabular}{|l|l|l|l|l|l|l|l|l|}
\hline \multicolumn{1}{|c|}{ Sample } & $\boldsymbol{B}\left(\mathbf{K}^{-\boldsymbol{a}}\right)$ & $\boldsymbol{\alpha}$ & $\boldsymbol{M}_{\boldsymbol{R} \boldsymbol{0}} / \boldsymbol{M}_{\boldsymbol{S} \boldsymbol{0}}$ & $\boldsymbol{T}_{\boldsymbol{O} \boldsymbol{R}}(\mathbf{K})$ & $\boldsymbol{\beta}$ & $\boldsymbol{\mu}_{\boldsymbol{0}} \boldsymbol{H}_{\boldsymbol{C} \boldsymbol{\theta}}(\mathbf{m} \mathbf{T})$ & $\boldsymbol{T}_{\boldsymbol{O C}}(\mathbf{K})$ & $\boldsymbol{m}$ \\
\hline $\begin{array}{l}\text { Nanowires array (H } \\
\text { applied parallel to Nws } \\
\text { axes) }\end{array}$ & $1.3 \times 10^{-5}$ & 1.4 & 0.63 & 1060 & 0.8 & 260 & 1289 & 2.5 \\
\hline $\begin{array}{l}\text { Core/shell nanowires } \\
\text { (released from the AAO } \\
\text { template) }\end{array}$ & $2.1 \times 10^{-3}$ & 0.9 & 0.62 & 470 & 1.9 & 405 & 389 & 1.1 \\
\hline
\end{tabular}

To further examine the saturation magnetization behavior with the temperature, the Bloch law was used to fit the $M_{S}$ vs. $T$ experimental data corresponding to nanowires into the AAO template. According to this law, the saturation magnetization can be modeled by [50]:

$\mathrm{M}_{\mathrm{S}}=\mathrm{M}_{\mathrm{S} 0}\left(1-\mathrm{BT}^{\alpha}\right)$

$M_{S O}$ is the saturation magnetization when temperature approaches to zero, $B$ is the Bloch constant, and $\alpha$ is the Bloch exponent. The $M_{S}$ parameters were obtained from the fits of approach law to magnetic saturation [51] (see insêt Fig. 4(â)).

The fitting results are shown in Fig. 5(a) and the obtained parameters are registered in Table 2. For the nanowires array $\alpha$ was 1.4 , which indicates that the nanostructure size is closer to the magnetic coherence length for canonical ferromagnetic materials [52]. Values of Bloch exponent of $3 / 2$ appears when the nanostructure size is larger than the single domain size. When the size is close to the magnetic coherence length, the dependence of $\alpha$ value with the nanostructure size is not clear [49]. On the other hand, there is an agreement on the dependence of the $B$ value with nanostructure size reduction [49]. A decrease of the nanostructure size leads to an increment of the Bloch constant, and 
the increment of the crystal disorder could be the main reason. One can notice such effect: for nanowires measured within the AOO template the estimated $B$ value is one magnitude order higher than the one obtained for $\mathrm{Fe}$ and $\mathrm{Co}$ in bulk $[53,54]$.

For $\mathrm{CoFe}_{2.7} / \mathrm{CoFe}_{2} \mathrm{O}_{4}$ core/shell nanowires, the $M_{S}$ parameters were extracted directly from the $M \mathrm{vs}$. $H$ curve (inset of Fig. 4(b)). When the Nws are partially oxidized is not possible to obtain an appropriate fit using equation (1). This is due to the superimposed phenomena emerging from each involved phase and because a more complex magnetization dynamics behavior probably takes place at the interface region between the $\mathrm{CoFe}_{2.7}$ core and the $\mathrm{CoFe}_{2} \mathrm{O}_{4}$ shell. Both facts make the system different from the canonical ferromagnetic behavior. To tackle this challenge, we employ the extended Bloch law (equation (2)) [55]. The extended law takes into account phenomena related to the nanostructure size and is given by:

$\mathrm{M}_{\mathrm{S}}=\mathrm{M}_{\mathrm{S} 0}\left(1-\mathrm{BT}^{\alpha}+\mathrm{A}_{0} \mathrm{e}^{\left(-\mathrm{T} / \mathrm{T}_{\mathrm{f}}\right)}\right)$

where $A_{0}$ and $T_{f}$ are experimental values typical of the material, depending on the nanostructure size [55]. The second term of the equation is related to surface disorder effects. Decoupled spins on surface could be responsible of the $M_{S}$ increment when temperature increases between 50 and $175 \mathrm{~K}$ (see Fig. 5(b)) [56]. It is worth noting that the Bloch constant for the core/shell systems is two orders of magnitude larger than those obtained for single phase nanowires (see Table 2). Such effect could be associated to several factors: increment of crystal disorder at the surface or interface, effects associated to the coexistence of two different magnetic phases [49], change in the average value of $J$ module [47], anisotropy effects [57], among others.

Fig. 5(c) shows the experimental data and the best fitting results (equation (3)) of the $M_{R} / M_{S} v s . T$ curves corresponding to single-phase and core/shell nanowires, respectively. The used model to fit the remanence magnetization is given by [58]:

$\frac{M_{R}}{M_{S}}=\frac{M_{R 0}}{M_{S 0}}\left[1-\left(\frac{T}{T_{0 R}}\right)^{\beta}\right]$

where $M_{R O} / M_{S O}$ is the reduced remanence without thermal fluctuation, and $T_{O R}$ is a characteristic temperature value depending on the material properties.

The value of $T_{O R}$ registered for nanowires into the AAO template is similar to the Curie temperature reported for iron-cobalt alloy $[59,60]$. When nanowires are released from the AAO template and are partially oxidized, to form core/shell architecture, $T_{O R}$ and $\beta$ values are modified (see Table 2). The $T_{O R}$ value decreases almost 50\%. Such temperature change could be associated with a change from a blocked magnetic state to a superparamagnetic state (the $\mathrm{CoFe}_{2} \mathrm{O}_{4}$ nanoparticle size is below the critical value [61]). The $\beta$ value changes significantly too. In the case of nanowires measured within the AAO template such value is similar to the expected for one-dimensional systems [44]. While for Nws released and partially oxidized, $\beta$ value is close to the reported for nanoparticles cluster [58]. This could be associated to the increment of the crystal disorder when the partial oxidation happens and two magnetic phases coexist.

Fig. 5(d) shows experimental values and the fitting of the $H_{C} / H_{C O}$ vs. $T$ curves. The Sharrock law [62] was used to fit the curves (equation (4)): 


\section{Conclusions}

A simple method for the synthesis of cobalt-iron/cobalt-ferrite bi-magnetic soft/hard magnetic core/shell nanowires is described. The proposed method is based in the oxidation at room conditions of $\mathrm{CoFe}_{2.7}$ nanowires obtained into the nanoporous AAO template. Electrodeposited nanowires are formed by $\mathrm{CoFe}$ and $\mathrm{CO}_{3} \mathrm{Fe}_{7}$ phases. The nanowires are formed by agglomerates of several nanoparticles in a compact array (with average nanoparticle size around $5 \mathrm{~nm}$ ). The nanostructures have a mean length between 1.2 and $3.7 \mu \mathrm{m}$, and diameters between 27 and $37 \mathrm{~nm}$ (aspect ratio from 32 to 137). When the nanowires were released from AAO template and partially oxidized, cobalt ferrite is formed in the surface. The magnetic properties of nanowires as a function of temperature were studied. For nanowires measured within the AAO template, results suggest a similar magnetic behavior to the reported for one-dimensional systems. Evidences indicate that the magnetic behavior of such nanowires is determined by cooperative effects of nanoparticles. The magnetic behavior changes when nanowires are released from the AAO template and are partially oxidized. A loss of cooperative effects in the magnetization mechanism was observed. In the same way, change from a blocked magnetic state to a superparamagnetic state (the $\mathrm{CoFe}_{2} \mathrm{O}_{4}$ nanoparticle size is below the 
critical value) was inferred. For the oxidized nanowires the magnetic behavior as a function of temperature is similar to the reported for nanoparticle clusters. This could be associated to the increment of the crystal disorder when the partial oxidation happens and two magnetic phases coexist.

\section{Acknowledgments}

This work was supported in part by the Agency of Argentine [CONICET PIP \#11220120100462]. The authors are grateful to C2NANO-Brazilian Nanotechnology National Laboratory (LNNano) at Centro Nacional de Pesquisa em Energìa e Materiais CNPEM/MCT (\#19927). O.M.-L. acknowledges FAPESP Grant 2014/26672-8.

\section{References}

[1] Tang J, Wang C Y, Hung M H, Jiang X, Chang L Te, He L, Liu P H, Yang HJ, Tuan H Y, Chen L J and Wang K L 2012 Ferromagnetic germanide in Ge nanowire transistors for spintronics application ACS Nano 6 5710-7

[2] Kim C H, Myung Y, Cho Y J, Kim H S, Park S H, Park J, Kim J Y and Kim B 2009 Electronic structure of Vertically aligned $\mathrm{Mn}$-doped $\mathrm{CoFe} 2 \mathrm{O} 4$ nanowires and their application as humidity sensors and photodetectors J. Phys. Chem. C $1137085-90$

[3] Wallentin J, Anttu N, Asoli D, Huffman M, Aberg I, Magnusson M H, Siefer G, FussKailuweit P, Dimroth F, Witzigmann B, Xu H Q, Samuelson L, Deppert K and Borgstrom M T 2013 InP Nanowire Array Solar Cells Achieving 13.8\{\%\} Efficiency by Exceeding the Ray Optics Limit Science (80-. ). 339 1057-60

[4] Wong W S, Raychaudhuri S, Lujan R, Sambandan S and Street R A 2011 Hybrid Si nanowire/amorphous silicon FETs for large-area image sensor arrays Nano Lett. 11 2214-8

[5] Felton E J and Reich D H 2007 Biological applications of multifunctional magnetic nanowires $11-22$

[6] Natelson D 2003 Fabrication of metal nanowires arXiv Prepr. cond-mat/0307600 1-16

[7] Prida V M, García J, Hernando B, Bran C, Vivas L G and Vázquez M 2015 Electrochemical synthesis of magnetic nanowires with controlled geometry and magnetic anisotropy

[8] Sellmyer D J, Zheng M and Skomski R 2001 Magnetism of Fe, Co and Ni nanowires in selfassembled arrays J. Phys. Condens. Matter 13 R433-60

[9] Zeng, H., Skomski, R., Menon, L., Liu, Y., Bandyopadhyay, S., Sellmyer, J. 2002 Structure and magnetic properties of ferromagnetic nanowires in self-assembled arrays Phys. Rev. B 65 $1344261-8$

[10] Zhou X, Liu S, Xu N, Wu Z and Huang J 2014 Facile route to preparation of Fe-Co microclusters with highly enhanced magnetic performances Mater. Lett. 136 325-8 
[11] Ciureanu M, Beron F, Clime L, Ciureanu P, Yelon A, Ovari T A, Cochrane R W, Normandin $\mathrm{F}$ and Veres T 2005 Magnetic properties of electrodeposited CoFeB thin films and nanowire arrays Electrochim. Acta $\mathbf{5 0} 4487-97$

[12] Arai K I, Kang H W and Ishiyama K 1991 Magnetic properties of Co-Fe electrodeposited alumite films IEEE Trans. Magn. 27 4906-8

[13] Reiss G and Hütten A 2005 Magnetic nanoparticles: Applications beyond data storage Nat. Mater. 4 725-6

[14] Yue G H, Wang X, Wang L S, Chang P, Wen R T, Chen Y Z and Peng D L 2009 Structure and magnetic properties of $\mathrm{Fe} 1-\mathrm{xC}$ Cx nanowires in self-assembled arrays Electrochim. Acta $\mathbf{5 4}$ 6543-7

[15] Ji R, Cao C, Chen Z and Yao R 2014 Synthesis of crystalline CoFex nanowire arrays through high voltage pulsed electrochemical deposition J. Magn. Magn. Mater.363 95-102

[16] Bran C, Palmero E M, Li Z-A, del Real R P, Spasova M, Farle M and Vázquez M 2015 Correlation between structure and magnetic properties in Cox Fe100- $\mathrm{x}$ nanowires: the roles of composition and wire diameter J. Phys. D. Appl. Phys. 48145304

[17] Qin D H, Cao L, Sun Q Y, Huang Y and Li H L 2002 Fine magnetic properties obtained in FeCo alloy nanowire arrays Chem. Phys. Lett. 358 484-8

[18] Qin D H, Peng Y, Cao L and Li H L 2003 A study of magnetic properties: FexCo1-x alloy nanowire arrays Chem. Phys. Lett. 374 661-6

[19] Ramazani A, Almasi Kashi M, Ghanbari S and Eshaghi F 2012 Dual behaviors of magnetic CoxFe1-x $(0<\mathrm{x}<1)$ nanowires embedded in nanoporous with different diameters J. Magn. Magn. Mater. 324 3193-8

[20] Sharif R, Shamaila S, Ma M, Yao L D, Yu R C, Han X F, Wang Y and Khaleeq-ur-Rahman M 2008 Magnetic and microstructural characterizations of $\mathrm{CoFe}$ and $\mathrm{CoFeB}$ nanowires $J$. Magn. Magn. Mater. $3201512-6$

[21] Ramazani A, Almasi-Kashi M, Golafshan E and Arefpour M 2014 Magnetic behavior of asdeposited and annealed $\mathrm{CoFe}$ and $\mathrm{CoFeCu}$ nanowire arrays by ac-pulse electrodeposition $J$. Cryst. Growth 402 42-7

[22] Germany S G 1996 Magnetic and structural properties of the electrochemically de- posited arrays of Co-Fe nanowires Thin Solid Films 27511429

[23] Lim S L, Xu F, Phuoc N N and Ong C K 2010 Length dependence of coercivity in CoFe2 nanowire arrays with high aspect ratios J. Alloys Compd. 505 609-12 
[24] Yang W, Cui C, Sun J and Wang B 2010 Fabrication and magnetic properties of Fe3Co7 alloy nanowire arrays J. Mater. Sci. $451523-7$

[25] Yue G H, Wang L S, Wang X, Chen Y Z and Peng D L 2009 Characterization and magnetic properties of Fe70Co30 alloy nanowire arrays J. Appl. Phys. 105074312

[26] Gao J H, Zhan Q F, He W, Sun D L and Cheng Z H 2006 Thermally activated magnetization reversal process of self-assembled Fe55Co45 nanowire arrays J. Magn. Magn. Mater. 305 $365-71$

[27] Su H L, Ji G B, Tang S L, Li Z, Gu B X and Du Y W 2005 Geometry dependence of the annealing effect on the magnetic properties of Fe 48 Co 52 nanowire arrays Nanotechnology 16 429-32

[28] Du H S and S T and R W and Y C and C J and Y 2009 Fe 48 Co 52 Alloy Nanowire Arrays: Effects of Magnetic Field Annealing Chinese J. Chem. Phys. 22 82-6

[29] Hua Z H, Chen R S, Li C L, Yang S G, Lu M, Gu B X and Du Y W 2007 CoFe2O4 nanowire arrays prepared by template-electrodeposition method and further oxidization J. Alloys Compd. 427 199-203

[30] Carlier D and Ansermet J-P 2006 Electrochemical Synthesis and Magnetic Properties of $\mathrm{CoFe}[$ sub 2]O[sub 4] Nanowire Arrays J. Electrochem. Soc. 153 C277

[31] Paterson B, Gyawali P, McKeown D, Buechele A, Pegg I L and Philip1 J 2013 Highly crystalline core-shell FeCo-CoFe2O4 nanostructures Eur. Phys. J. Appl. Phys. 63 30401(6)

[32] López-Ortega A, Estrader M,Salazar-Alvarez G, Roca A G and Nogués J 2015 Applications of exchange coupled bi-magnetic hard/soft and soft/hard magnetic core/shell nanoparticles Phys. Rep. 553 1-32

[33] Londoño-Calderón C L, Biolovol V, Cosio-Catañeda C, Pampillo L G, Micheli S R, Pirota K R, Socolovsky L M and Martínez-García R 2013 Synthesis and Characterization of Iron Oxyhydroxide Nanowires IEEE Trans. Magn. 49 4502-5

[34] Furneaux R C, Rigby W R and Davidson A P 1989 The formation of controlled-porosity membranes from anodically oxidized aluminium Nature 337 147-9

[35] Lourakis M I a 2005 A Brief Description of the Levenberg-Marquardt Algorithm Implemened by levmar Matrix 32

[36] Wang C M, Baer D R, Thomas L E, Amonette J E, Antony J, Qiang Y and Duscher G 2005 Void formation during early stages of passivation: Initial oxidation of iron nanoparticles at room temperature J. Appl. Phys. 98 094308-7 
[37] Mourdikoudis S, Simeonidis K, Angelakeris M, Tsiaoussis I, Kalogirou O, Desvaux C, Amiens C and Chaudret B 2007 Effect of Air Exposure on Structural and Magnetic Features of FeCo Nanoparticles Mod. Phys. Lett. B 21 1161-8

[38] Zhang J M, Wang D D and Xu K W 2006 Calculation of the surface energy of bcc transition metals by using the second nearest-neighbor modified embedded atom method Appl. Surf. Sci. $2528217-22$

[39] Turgut $\mathrm{Z}$ and H 2009 Isothermal oxidation behaviors of FeCoV and FeCoVNballoys J.Appl. Phys. 105 07A330-3

[40] Jones N J, McNerny K L, Wise A T, Sorescu M, McHenry M E and Laughlin D E 2010 Observations of oxidation mechanisms and kinetics in faceted FeCo magnetic nanoparticles $J$. Appl. Phys. 107 09A304-3

[41] Turgut Z, Huang M-Q, Gallagher K, McHenry M E and Majetich S a.1997 Magnetic evidence for structural-phase transformations in Fe-Co alloy nanocrystals produced by a carbon arc J. Appl. Phys. 81 4039-41

[42] Kuhrt C and Schultz L 1992 Formation and magnetic properties of nanocrystalline mechanically alloyed Fe-Co J. Appl. Phys. 71 1896-900

[43] Schlörb H, Uhlemann M, Haehnel V, Iselt D and Gebert A 2013 Electrodeposition of Febased Magnetic Alloy Nanowires Zeitschrift für Phys. Chemie 227130603060405002

[44] Franco V and Conde A 2004 Thermal effects in a Stoner-Wohlfarth model and their influence on magnetic anisotropy determination J. Magn. Magn. Mater. 278 28-38

[45] Roberts A P, Cui Y and Verosub K L 1995 Wasp-waisted hysteresis loops: Mineral magnetic characteristics and discrimination of components in mixed magnetic systems J. Geophys. Res. 100 17909-24

[46] Tauxe L, Mullender T a. T and Pick T 1996 Potbellies, wasp-waists, and superparamagnetism in magnetic hysteresis J. Geophys. Res. 101 571-83

[47] Sperl M, Kipferl W, Dumm M and Bayreuther G 2006 Spin-wave excitations in epitaxial ultrathin FeCo with zero magnetocrystalline anisotropy J. Appl. Phys. 99 08J703-3

[48] Srivastava C M, Srinivasan G and Nanadikar N G 1979 Exchange constants in spinel ferrites Phys. Rev. B 19 499-508

[49] Zhang D, Klabunde K, Sorensen C and Hadjipanayis G 1998 Magnetization temperature dependence in iron nanoparticles Phys. Rev. B 58 14167-70 
[50] Aquino R, Depeyrot J, Sousa M H, Tourinho F A, Dubois E and Perzynski R 2005 Magnetization temperature dependence and freezing of surface spins in magnetic fluids based on ferrite nanoparticles Phys. Rev. B - Condens. Matter Mater. Phys. 72 184435-10

[51] Brown W 1940 Theory of the approach to magnetic saturation Phys. Rev. 736-43

[52] Herring C and Kittel C 1951 On the theory of spin waves in ferromagnetic media Phys. Rev. $81869-80$

[53] Koksharov Y A 2009 Magnetism of Nanoparticles: Effects of Size, Shape, and Interactions ed S P Gubin (Weinheim, Germany: Wiley-VCH Verlag GmbH \& Co.)

[54] Wu M, Zhang Y D, Hui S, Xiao T D, Ge S, Hines W a. and Budnick J I 2004 Temperature dependence of magnetic properties of SiO2-coated Co nanoparticles J. Magn. Magn. Mater. $26820-3$

[55] Vázquez-Vázquez C, López-Quintela M A, Buján-Núñez M C and Rivas J 2011 Finite size and surface effects on the magnetic properties of cobalt ferrite nanoparticles J. Nanoparticle Res. 13 1663-76

[56] Vazquez-Vazquez C, Lovelle M, Mateo C, Loez-Quintela M, Bujan-Nunez M, Serantes D, Baldomir D and Rivas J 2008 Magnetocaloric effect and size-dependent study of the magnetic properties of cobalt ferrite nanoparticles prepared by solvothermal synthesis Phys. Status Solidi a-Applications Mater. Sci. 205 1358-62

[57] Bayreuther G 1989 Magnetic surfaces Hyperfine Interact. 47-48 237-49

[58] Wang J, Duan H, Lin X, Aguilar V, Mosqueda A and Zhao G M 2012 Temperature dependence of magnetic anisotropy constant in iron chalcogenide Fe3Se4: Excellent agreement with theories J. Appl. Phys. 112 103905-5

[59] Kawahara K, Iemura D, Tsurekawa S and Watanabe T 2003 High Temperature In-situ Observations of Magnetic Domains in Fe-Co Alloys Mater. Trans. 44 2570-7

[60] Ohnuma I, Enoki H, Ikeda O, Kainuma R, Ohtani H, Sundman B and Ishida K 2002 Phase equilibria in the Fe-Co binary system Acta Mater. 50 379-93

[61] Menchaca-Nal S, Londoño-Calderón C L, Cerrutti P, Foresti M L, Pampillo L, Bilovol V, Candal R and Martínez-García R 2016 Facile synthesis of cobalt ferrite nanotubes using bacterial nanocellulose as template Carbohydr. Polym. 137 726-31

[62] Sharrock M P 1994 Time dependence of switching fields in magnetic recording media (invited) J. Appl. Phys. 76 6413-8 
[63] Victora R H 1989 Predicted time dependence of the switching field for magnetic materials Phys. Rev. Lett. 63 457-60

[64] Wernsdorfer W 2001 Classical and quantum magnetization reversal studied in nanometersized particles and clusters Adv. Chem. Phys. 11894

[65] Torres T E, Lima Jr. E, Mayoral A, Ibarra A, Marquina C, Ibarra M R and Goya G F 2015 Validity of the Néel-Arrhenius model for highly anisotropic CoxFe3-xO4 nanoparticles $J$. Appl. Phys. 118183902

[66] Maurer T, Zighem F, Fang W, Ott F, Chaboussant G, Soumare Y, Atmane K A, Piquemal J Y and Viau G 2011 Dipolar interactions in magnetic nanowire aggregates J. Appl. Phys. 110 $123924-6$

[67] Fang W, Panagiotopoulos I, Ott F, Boué F, Ait-Atmane K, Piquemal J Y, Viau G and Dalmas F 2014 Optimization of the magnetic properties of aligned Co nanowires/polymer composites for the fabrication of permanent magnets J. Nanoparticle Res. 16

[68] Zighem F, Maurer T, Ott F and Chaboussant G 2011/Dipolar interactions in arrays of ferromagnetic nanowires: A micromagnetic study J. Appl. Phys. 109 013910-8

[69] Karanasos V, Panagiotopoulos I, Niarchos D, Okumura H and Hadjipanayis G C 2001 CoPt:B granular thin films for high density magnetic recording media J. Magn. Magn. Mater. 236 $234-41$

[70] Givord D, Tenaud P and Vladieu T 1988 Coercivity mechanisms in ferrite and rare earth transition metal sintered magnets (SmCo5, Ns-Fe-B) IEEE Trans. Magn. 24 1921-3 